\title{
Single-top $W t$-channel production matched with parton showers using the POWHEG method
}

\author{
Emanuele $\operatorname{Re}^{\mathrm{a}}$ \\ Institute for Particle Physics Phenomenology, Department of Physics, University of Durham, Durham, DH1 3LE, UK
}

Received: 3 November 2010 / Revised: 9 December 2010 / Published online: 3 February 2011

(C) The Author(s) 2011. This article is published with open access at Springerlink.com

\begin{abstract}
We present results for the next-to-leading order calculation of single-top $W t$-channel production interfaced to Shower Monte Carlo programs, implemented according to the POWHEG method. A comparison with MC@NLO is carried out. Results obtained using the PYTHIA shower are also shown and the effect of typical cuts is briefly discussed.
\end{abstract}

\section{Introduction}

Top-quark production is one of the most important processes at hadron colliders. Within the Standard Model, top quarks can be produced in pairs (via strong interaction) or individually (via electroweak processes). Top-antitop pair production is known to have the largest cross section, and has been studied extensively, both experimentally and theoretically. In fact, the top quark was discovered in 1995 at the Tevatron in $t \bar{t}$ production events. The observation of single-top electroweak production is instead more difficult and it was announced by the Tevatron experiments more recently $[1,2]$. This was due not only to the fact that the single-top cross section is smaller than the $t \bar{t}$ one, but also to the presence of large backgrounds, namely $W+$ jet and $t \bar{t}$, that required highly non-trivial analysis strategies.

Although experimentally challenging, single-top electroweak production is particularly important since it provides a relatively clean place to study the electroweak properties of the top quark. For instance, it allows for a direct measurement of the $V_{t b}$ CKM matrix element [3], which is important in testing the unitarity of the CKM matrix. Moreover, electroweak-produced top quarks are highly polarized, hence angular correlations of top-quark decay products are particularly sizeable $[4,5]$, providing a good probe of the spin structure of the $W-t-b$ vertex. Finally, since the topquark mass is close to the scale of electroweak symmetry breaking, new physics effects could be discovered in

a e-mail: emanuele.re@durham.ac.uk the top-quark sector, and reactions where only a single-top is produced are particularly sensitive to some BSM models (see, for example, $[6,7])$ ). Single-top production (in the $W t$-channel) is also an important background for some Higgs-boson search channels, such as $H \rightarrow W^{+} W^{-}$[8].

The hadroproduction of single-top quarks in protonproton collisions is traditionally classified according to the partonic processes present at LO: the s-channel processes $(q \bar{q} \rightarrow t b)$ involve the exchange of a time-like $W$ boson, the $t$-channel processes $\left(b q \rightarrow t q^{\prime}\right)$ involve the exchange of a space-like $W$ boson, while associated $W t$ production ( $b g \rightarrow t W^{-}$) involves the production of a top quark in association with a $W$ boson. The $t$-channel process is the main source of single-top quarks, both at the Tevatron and the LHC. At the Tevatron the $W t$ contribution is negligible and the $s$-channel cross section is roughly half of the $t$ channel's one. At the LHC, instead, the $W t$-production cross section is a factor 3 less than the $t$-channel's one, while the $s$-channel is negligible. Therefore, an accurate description of all the three production channels is important: in particular, at the LHC, the $W t$-channel will play a significant role.

Given the above reasons, it is then desirable to reach a high precision in the theoretical predictions, both for total rates and for more exclusive distributions. Higher-order corrections to single-top hadroproduction have been calculated in [9-20]. Nowadays, one of the ways to go beyond this level of accuracy is to merge the fixed-order accuracy of a NLO calculation with the (Next-to)-LeadingLogarithm accuracy (and the flexibility) of a (parton shower) Monte Carlo event generator. At present there are two methods to interface NLO calculations with parton showers in a consistent way: MC@NLO [21] and POWHEG [22, 23]. ${ }^{1}$ They have been applied to several processes at lepton [27-29]

\footnotetext{
${ }^{1}$ In literature, other proposals exist [24-26]. The MC@NLO and POWHEG methods are the only ones where, currently, several full processes at lepton and hadron colliders have been implemented.
} 
and hadron [30-44] colliders, in conjunction with the HERWIG [45], PYTHIA [46] and HERWIG++ [47] parton shower algorithms. ${ }^{2,3}$ Despite the theoretical formulation of the two methods being quite different, reasonable agreement has been found as well as the reason for the (few) differences encountered (see for example [50] for a recent discussion).

In [41] the details of the implementation of single-top $s$ - and $t$-channel production processes in the POWHEG framework have already been described. The aim of this paper is to describe the implementation of the $W t$-channel production process. Therefore, results presented here can be seen as a completion of the work described in the previous reference.

This paper is organized as follows. In Sect. 2 we describe some of the technicalities of the implementation. We also summarize how the problem of the interference between the NLO corrections to this process and the $t \bar{t}$ calculation has been dealt with. Here we anticipate that we have used the same strategy adopted by the MC@NLO authors, i.e. two definitions have been implemented, such that their difference can be considered a measure of one of the theoretical uncertainties that affects this process. In Sect. 3 results are presented: in particular we compare POWHEG and MC@NLO results for the two aforementioned definitions of the NLO corrections. We also show results obtained with typical cuts and results obtained using the PYTHIA shower. Finally, in Sect. 4 we give our conclusions.

\section{The POWHEG implementation}

To match single-top $W t$-channel production NLO corrections with a parton shower using the POWHEG method, the POWHEG-BOX package has been used. POWHEG-BOX is a program that automates all the steps described in [23], turning a NLO calculation into a POWHEG simulation. The details of how the program works have been largely described in [51]. In practice, starting from some of the typical building blocks of the NLO calculation for the process at hand, the program produces a set of partonic events ready to be showered by a shower Monte Carlo program.

In this section we present some of the inputs that we calculated and then coded in the format needed by the POWHEG-BOX package to work. In particular, we describe how the LO kinematics and the relevant differential cross sections up to next-to-leading order in the strong coupling

\footnotetext{
${ }^{2}$ In [48] a compared study of the effects of different parton shower algorithms has also been performed, in case of Higgs-boson production via gluon fusion.

${ }^{3}$ An implementation of the POWHEG method has also appeared recently within the SHERPA event generator [49].
}

$\alpha_{\mathrm{S}}$ have been obtained. All quark masses have been set to zero (except, of course, the top-quark mass) and the full Cabibbo-Kobayashi-Maskawa (CKM) matrix has been taken into account in the calculation. However, for sake of illustration, through this section we set the CKM matrix equal to the identity. Therefore, the $d$-type quark connected to the top and the $W$ boson will be denoted as $b$. Furthermore, in this paper we always refer to top-quark production: antitop production is obtained simply by charge conjugation.

With this convention, the LO partonic process for $W t$ production is

$b+g \rightarrow W^{-}+t$

We denote with $\mathcal{B}_{b g}$ the (summed and averaged) squared amplitude, divided by the flux factor. The process initiated by the partons $g b$ is also present. For brevity, throughout the paper processes that can be obtained from the written ones by simply exchanging the order of the incoming partons will be omitted from the formulae.

Before giving the relevant formulae, here we stress again that the NLO corrections to the $W t$ production channel are not well defined, due to interference effects with the $t \bar{t}$ process. This problem is well known, and several approaches have been introduced $[15,52,53]$. To deal with this theoretical issue, we used the same strategy as first described in the corresponding MC@NLO publication [36] and later extensively studied in [54]: two definitions for the NLO corrections have been considered, and the relative difference between the two can be interpreted as a measure of the theoretical uncertainty in the definition of genuine NLO corrections to the $W t$-channel process. Since this issue concerns the radiative part of the QCD NLO corrections, a more accurate discussion will be given in Sect. 2.3.

\subsection{Born kinematics}

Following the notation of [23], we denote with $\bar{k}_{\oplus}$ and $\bar{k}_{\ominus}$ the incoming parton momenta, aligned along the plus and minus direction of the $z$ axis, and by $\bar{k}_{1}$ and $\bar{k}_{2}$ the outgoing $W$-boson and top-quark momenta, respectively. The topquark and $W$-boson masses are denoted by $m_{t}$ and $m_{W}$. During the POWHEG-algorithm step where the inclusive NLO cross section is evaluated (the calculation of the $\bar{B}$ function), their virtualities are kept fixed and equal to their masses. If $K_{\oplus}$ and $K_{\ominus}$ are the momenta of the incoming hadrons, then we have

$\bar{k}_{\oplus}=\bar{x}_{\oplus} K_{\oplus}$,

where $\bar{x}_{\oplus}$ are the momentum fractions, and momentum conservation reads

$\bar{k}_{\oplus}+\bar{k}_{\ominus}=\bar{k}_{1}+\bar{k}_{2}$. 
We now introduce the variables

$\bar{s}=\left(\bar{k}_{\oplus}+\bar{k}_{\ominus}\right)^{2}$,

$\bar{Y}=\frac{1}{2} \log \frac{\left(\bar{k}_{\oplus}+\bar{k}_{\ominus}\right)^{0}+\left(\bar{k}_{\oplus}+\bar{k}_{\ominus}\right)^{3}}{\left(\bar{k}_{\oplus}+\bar{k}_{\ominus}\right)^{0}-\left(\bar{k}_{\oplus}+\bar{k}_{\ominus}\right)^{3}}$,

and $\bar{\theta}$, the angle between the outgoing top quark and the $\bar{k}_{\oplus}$ momentum, as seen in the partonic center-of-mass (CM) frame. We denote with $\bar{\phi}$ the azimuthal angle of the outgoing top quark in the same reference frame. Since the differential cross sections do not depend on the overall azimuthal orientation of the outgoing partons, we set this angle to zero. At the end of the generation of an event, we perform a uniform, random azimuthal rotation of the whole event, in order to cover the whole final-state phase space. The set of variables $\overline{\boldsymbol{\Phi}}_{2} \equiv\{\bar{s}, \bar{Y}, \bar{\theta}, \bar{\phi}\}$ fully parameterizes the Born kinematics. From them, we can reconstruct the momentum fractions

$\bar{x}_{\oplus}=\sqrt{\frac{\bar{s}}{S}} e^{\bar{Y}}, \quad \bar{x}_{\ominus}=\sqrt{\frac{\bar{s}}{S}} e^{-\bar{Y}}$

where $S=\left(K_{\oplus}+K_{\ominus}\right)^{2}$ is the squared CM energy of the hadronic collider. The outgoing momenta are first reconstructed in their longitudinal rest frame, where $\bar{Y}=0$. In this frame, their energies are

$\left.\bar{k}_{1}^{0}\right|_{\bar{Y}=0}=\frac{\bar{s}+m_{W}^{2}-m_{t}^{2}}{2 \sqrt{\bar{s}}}$,

$\left.\bar{k}_{2}^{0}\right|_{\bar{Y}=0}=\frac{\bar{s}+m_{t}^{2}-m_{W}^{2}}{2 \sqrt{\bar{s}}}$.

The two spatial momenta are opposite and their modulus $|\mathbf{k}|_{\bar{Y}=0}$ is found by using the on-shell condition $m_{W}^{2}=$ $\left(\left.\bar{k}_{1}^{0}\right|_{\bar{Y}=0}\right)^{2}-\left(|\mathbf{k}|_{\bar{Y}=0}\right)^{2}$. We fix the top-quark momentum to form an angle $\bar{\theta}$ with the $\oplus$ direction and to have zero azimuth (i.e. it lies in the $x z$ plane and has positive $x$ component). Both $\bar{k}_{1}$ and $\bar{k}_{2}$ are then boosted back in the laboratory frame, with boost rapidity $\bar{Y}$. The Born phase space, in terms of these variables, can be written as

$$
\begin{aligned}
d \overline{\boldsymbol{\Phi}}_{2}= & d \bar{x}_{\oplus} d \bar{x}_{\ominus}(2 \pi)^{4} \delta^{4}\left(\bar{k}_{\oplus}+\bar{k}_{\ominus}-\bar{k}_{1}-\bar{k}_{2}\right) \\
& \times \frac{d^{3} \bar{k}_{1}}{(2 \pi)^{3} 2 \bar{k}_{1}^{0}} \frac{d^{3} \bar{k}_{2}}{(2 \pi)^{3} 2 \bar{k}_{2}^{0}} \\
= & \frac{1}{S} \frac{\beta}{16 \pi} d \bar{s} d \bar{Y} d \cos \bar{\theta} \frac{d \bar{\phi}}{2 \pi}
\end{aligned}
$$

where

$$
\begin{aligned}
& \beta=\sqrt{1-\rho}, \\
& \rho=\frac{2\left(m_{W}^{2}+m_{t}^{2}\right)}{\bar{s}}-\frac{\left(m_{W}^{2}-m_{t}^{2}\right)^{2}}{\bar{s}^{2}} .
\end{aligned}
$$

The content of (7) and the procedure described above to define the parton momenta from the variables set $\overline{\boldsymbol{\Phi}}_{2}$ have been coded in the subroutine born_phsp of the POWHEG-BOX package [51].

\subsection{Born and virtual contributions}

The squared matrix element for the lowest-order subprocess $b g \rightarrow W^{-} t$ has been obtained with MadGraph [55]. It has been checked with the expression reported in (3.5) of [36] and with an independent calculation performed with FeynCalc [56], starting from the two Feynman diagrams in Fig. 1. The top-quark width $\Gamma_{t}$ has been set here to zero.

To regularize soft and collinear divergences, the POWHEG-BOX package uses an automated implementation of the subtraction algorithm proposed by Frixione, Kunszt and Signer [57, 58]. The counterterms needed for the numerical subtraction of the real squared amplitudes soft divergences are obtained from the colour-linked Born squared amplitudes $\mathcal{B}_{i j}$ defined in (2.97) of [23], using the eikonal approximation. For the process at hand, the $\mathcal{B}_{i j}$ matrix is proportional to the full Born squared amplitude. In fact, since at the lowest order we have only three colourconnected partons, the equality

$\mathbf{T}_{\oplus}+\mathbf{T}_{\ominus}+\mathbf{T}_{2}=0$

holds, where $\mathbf{T}_{i}$ is the colour operator associated with the $i$ parton in the Born process (1). Using the property

$\mathbf{T}_{i} \cdot \mathbf{T}_{j}=\frac{\left(\mathbf{T}_{i}+\mathbf{T}_{j}\right)^{2}-\mathbf{T}_{i}^{2}-\mathbf{T}_{j}^{2}}{2}$

and the fact that $\mathbf{T}_{i}^{2}=C_{\mathrm{F}}$ if $i$ is a quark and $\mathbf{T}_{i}^{2}=C_{\mathrm{A}}$ if $i$ is a gluon, we obtain $\mathcal{B}_{i j}=\left(2 C_{\mathrm{F}}-C_{\mathrm{A}}\right) \mathcal{B}_{b g} / 2$ if neither $i$ or $j$ are gluons, and $\mathcal{B}_{i j}=C_{\mathrm{A}} \mathcal{B}_{b g} / 2$ if $i$ or $j$ is a gluon.

Collinear counterterms are obtained using the collinear factorization. However, since in the $W t$-channel LO process (1) an external gluon is present, the collinear limits associated with this leg do not factorize in terms of the Altarelli-Parisi unpolarized splitting kernels times the Born contribution $\mathcal{B}_{b g}$. In fact azimuthal correlations in the branching process are present, and to build a local counterterm the POWHEG-BOX package makes use of the spincorrelated Born cross sections $\mathcal{B}_{\mu \nu}$, defined in (2.8) of [23].

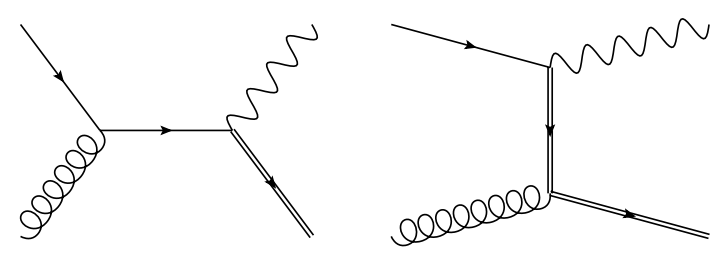

Fig. 1 Feynman diagrams for the LO process $b g \rightarrow W^{-} t$. The top quark is denoted with a double line 
The FeynCalc program has been used to calculate this matrix and to translate the result in a Fortran routine.

One-loop virtual contributions have been calculated and algebraically reduced to scalar integrals using the Passarino-Veltman algorithm. We used the same renormalization procedure described in [36]. We checked that the IR pole structure we are left with corresponds to the singularities of the real contributions. The finite part that enters in the soft-virtual contribution $\mathcal{V}_{b g}((4.6)$ of [51]) is computed numerically, using the package QCDloop [59] to evaluate the finite part of the scalar integrals.

\subsection{Real contributions}

The real emission corrections can be classified as follows:

$b+g \rightarrow W^{-}+t+g$,

$b+q(\bar{q}) \rightarrow W^{-}+t+q(\bar{q}) \quad(q \neq b)$,

$g+g \rightarrow W^{-}+t+\bar{b}$,

$q+\bar{q} \rightarrow W^{-}+t+\bar{b}$.

We denote the corresponding contributions to the cross section as $\mathcal{R}_{b g}, \mathcal{R}_{b q}, \mathcal{R}_{g g}$ and $\mathcal{R}_{q \bar{q}}$, respectively, where we used again the standard POWHEG notation first introduced in [23]. The 3-body phase space is denoted as $\boldsymbol{\Phi}_{3}$ and the corresponding momenta as $k_{\oplus}, k_{\ominus}, k_{1}, k_{2}$ and $k_{3}$, where $k_{3}$ is the momentum of the outgoing light parton (the FKS parton) while the other momenta correspond to those of the two incoming partons, the $W$-boson and the top quark. In Fig. 2 some representative diagrams are shown.

The processes (13) and (14) have a final state that corresponds to $t \bar{t}$ production followed by the decay $\bar{t} \rightarrow W^{-} \bar{b}$. A consequence of this fact is the well known problem of interference between $W t$ and $t \bar{t}$ production and it is the reason

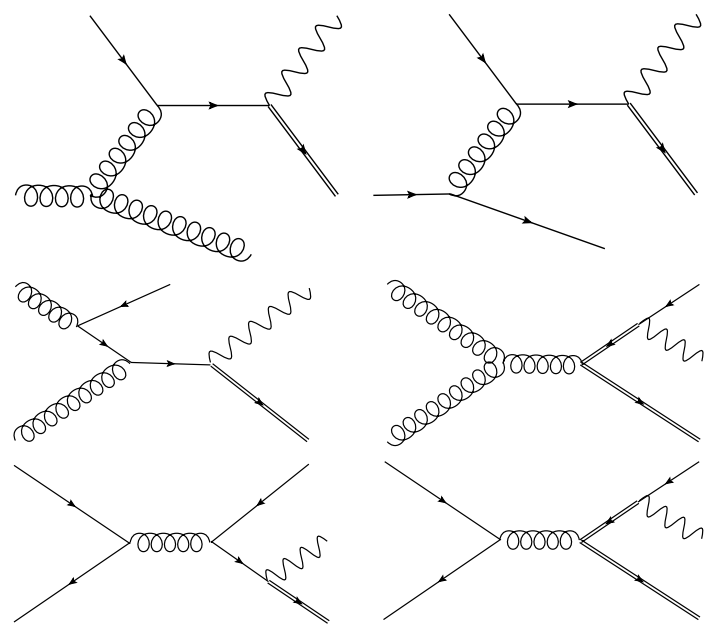

Fig. 2 Representative Feynman diagrams for the processes (11), (12) (up), (13) (center) and (14) (bottom) why QCD NLO corrections to the $W t$-channel are not well defined. In the following we will state more precisely the nature of the problem and explain how we dealt with it.

As one can see from the last four Feynman diagrams in Fig. 2, diagrams associated with the subprocesses (13) and (14) can be divided into two sets. Following the nomenclature of [36], we call doubly resonant the diagrams where a top-quark propagator goes on-shell when the invariant mass of the system made by the $W$-boson and the outgoing $\bar{b}$-quark $\left(m_{W \bar{b}}\right)$ approaches $m_{t}$. The other diagrams we call singly resonant. Since these two sets of diagrams have to be summed at the amplitude level, interference effects are present. This interference between NLO real corrections to $W t$ and lowest-order $t \bar{t}$ production (followed by a decay) would not be a problem if the contamination on $t \bar{t}$-like diagrams was numerically negligible with respect to the size of singly resonant diagrams. This is certainly not the case when one approaches the region $\left(k_{W}+k_{\bar{b}}\right)^{2} \equiv\left(k_{1}+k_{3}\right)^{2} \sim m_{t}^{2}$. In fact, in this region the real corrections to doubly resonant diagrams become huge (an internal propagator goes on-shell), and the perturbative expansion (in power of $\alpha_{\mathrm{S}}$ ) for the NLO corrections to single-top $W t$-channel loses its meaning.

To deal with this problem, several approaches are possible:

- The more drastic approach is to consider that top quarks are not detectable particles. This approach would remove any interference issue, since processes would be unambiguously classified accordingly only to experimentally measurable (QCD) final states: one would have the processes $W^{+} W^{-} b \bar{b}$ and $W^{-} W^{+} b$. At present, the price to pay would be to neglect NLO corrections, since these are not available for processes where top quarks are not on-shell. ${ }^{4}$

- An alternative approach is to give a prescription for removing the contamination from $t \bar{t}$-like contributions, while keeping the top quark as a final-state particle. This task can be accomplished using cuts to avoid the doubly resonant region or including an ad hoc subtraction term to remove (or suppress) the $t \bar{t}$ contribution.

The cut strategy was first adopted in [52], where an explicit cut on $m_{W \bar{b}}$ was used. In [15] a $b$-jet veto was instead used, together with a careful choice of the factorization scale. Moreover, in [15] for the first time differential results at the NLO were presented and QCD corrections to the top-quark decay have been also included, in the narrow-width approximation.

\footnotetext{
${ }^{4}$ We also recall that a calculation of NLO corrections to the production and the decay of top quarks performed in the narrow width approximation (as the one appeared in [15]) would not avoid the interference problem. To our knowledge, the only calculations that fully include top off-shellness effects are the ones described in $[60,61]$ for single-top $s$ - and $t$-channel production, respectively.
} 
In [53] a subtraction term was first introduced in the context of an inclusive $\mathrm{LO}(+\mathrm{LL})$ calculation. A similar method has been adopted in the fully inclusive NLO calculation presented in [13].

Finally, in $[36,54]$ the interference problem was reexamined at length, and it was shown that a separate treatment of $t \bar{t}$ and $W t$ production is feasible, also when the NLO calculation is matched with a parton shower.

In this work we have used the same approach described by the MC@NLO authors. This relies on the observation that a meaningful definition of the $W t$-channel process (as a signal or a background) is possible only through cuts on finalstate objects. If interference effects with $t \bar{t}$ are negligible after these cuts are applied, then it is possible to consider $W t$-channel a well-defined process. Since cuts act differently in different phase-space regions, one needs to quantify the interference between $W t$ and $t \bar{t}$ locally in the phase space. To this end, two definitions for the NLO corrections were given. It was shown that by comparing the two results it is possible to address the previous question, and it was found that, for several sets of cuts, the theoretical uncertainty due to interference effects is typically negligible with respect to other theoretical errors. In the following we will discuss briefly the two subtraction strategies and how they have been implemented within the POWHEG framework. In Sect. 3 the corresponding results will be shown.

The two definitions proposed in [36] are known as Diagram Removal (DR) and Diagram Subtraction (DS). Their difference can be better understood by writing a generic amplitude for the processes (13) and (14) as

$\mathcal{M}=\mathcal{M}^{W t}+\mathcal{M}^{t \bar{t}}$,

where $\mathcal{M}^{W t}$ and $\mathcal{M}^{t \bar{t}}$ denote, respectively, the sum of all the singly and doubly resonant Feynman diagrams for the partonic subprocess at hand. In DR one defines the real contribution $\mathcal{R}$ by eliminating the $t \bar{t}$ contribution $\mathcal{M}^{t \bar{t}}$ from $\mathcal{M}$ before squaring the amplitude. Instead in DS one keeps the full squared amplitude but subtracts from it a local counterterm $\mathcal{C}^{\mathrm{SUB}}$ in order to suppress the $t \bar{t}$ contribution at the cross section level. In this respect, DS can be seen as a refinement of the method proposed in [53]. Schematically, we have

$\mathcal{R}^{\mathrm{DR}}=\frac{\left|\mathcal{M}^{W t}\right|^{2}}{2 s}$,

$\mathcal{R}^{\mathrm{DS}}=\frac{\left|\mathcal{M}^{W t}+\mathcal{M}^{t \bar{t}}\right|^{2}-\mathcal{C}^{\mathrm{SUB}}}{2 s}$,

where $s$ is the squared CM energy. Some comments are due here.

- The DR method is not gauge invariant. This issue was studied in depth by the authors of [36], and it was shown that the impact of gauge dependence in the DR calculation is numerically negligible.
- In the DS approach one wants to build a gauge invariant subtraction term that exactly cancels the $t \bar{t}$ contribution when the doubly resonant region is approached. Thus, the subtraction term $\mathcal{C}^{\text {SUB }}$ has to fulfill the following requirements:

1. gauge invariance;

2. match exactly the $\left|\mathcal{M}^{t \bar{t}}\right|^{2}$ contribution when the doubly resonant region is approached;

3. possibly fall off (quickly) far from the doubly resonant region.

The third requirement is needed to keep the full NLO corrections unmodified away from the $t \bar{t}$ peak. Apart from the three requirements above, there is some freedom in the definition of $\mathcal{C}^{\mathrm{SUB}}$.

- By taking the difference between (17) and (16), one finds

$$
\mathcal{R}^{\mathrm{DS}}-\mathcal{R}^{\mathrm{DR}}=\frac{\mathcal{I}+\left|\mathcal{M}^{t \bar{t}}\right|^{2}-\mathcal{C}^{\mathrm{SUB}}}{2 s},
$$

where $\mathcal{I}=2 \Re\left(\mathcal{M}^{W t} \mathcal{M}^{t t^{*}}\right)$. Therefore, the difference between results obtained with DR and DS can be interpreted as a measure of the size of the interference $\mathcal{I}$, provided that the difference $\left|\mathcal{M}^{t \bar{t}}\right|^{2}-\mathcal{C}^{\mathrm{SUB}}$ is small.

We implemented in POWHEG the two subtraction methods. The squared amplitudes $|\mathcal{M}|^{2}$ and $\left|\mathcal{M}^{W t}\right|^{2}$ have been obtained using MadGraph. The subtraction term was chosen as in [36]:

$$
\begin{aligned}
& \mathcal{C}^{\mathrm{SUB}}\left(\boldsymbol{\Phi}_{3}\right) \\
& =\frac{\left(m_{t} \Gamma_{t}\right)^{2}}{\left(\left(k_{1}+k_{2}\right)^{2}-m_{t}^{2}\right)^{2}+\left(m_{t} \Gamma_{t}\right)^{2}}\left|\mathcal{M}^{t \bar{t}}\left(\boldsymbol{\Phi}_{3}^{\prime}\right)\right|^{2},
\end{aligned}
$$

where $\boldsymbol{\Phi}_{3}^{\prime}$ is a point in the 3-body phase space obtained by reshuffling the $\boldsymbol{\Phi}_{3}$ kinematics in order to have $\left(k_{1}+k_{3}\right)^{2}=$ $m_{t}^{2}$, i.e. an exactly doubly resonant configuration. Hence, the choice of the subtraction term is the same as the one used by the MC@NLO authors. In fact, in spite of the aforementioned freedom, as was already pointed out in [36], the choice of the amplitude $\mathcal{M}^{t \bar{t}}$ evaluated at the point $\boldsymbol{\Phi}_{3}^{\prime}$ is unavoidable if one wants to achieve the exact cancellation of the doubly resonant contribution while retaining gauge invariance, since $\mathcal{M} \sim \mathcal{M}^{t \bar{t}}$ when $\left(k_{1}+k_{3}\right)^{2} \rightarrow m_{t}^{2}$ and gauge invariance is preserved only if the internal $\bar{t}$ propagator is on-shell. The only real freedom is in the choice of the prefactor, and the Breit-Wigner profile seems the more natural choice if one wants the difference between $\left|\mathcal{M}^{t \bar{t}}\right|^{2}$ and $\mathcal{C}^{\mathrm{SUB}}$ to be close to zero as much as possible away from the resonance.

The inclusion of DR in POWHEG is straightforward. The procedure we adopted for DS is more subtle, since $\mathcal{R}^{\mathrm{DS}}$ is not positive-definite. This affects two steps of the POWHEG method. In the following we describe how we proceeded in our implementation. As usual, we use the standard notation of [23]. 
- Having a real correction that is not always positivedefinite increases the chances to have regions of $\overline{\boldsymbol{\Phi}}_{2}$ where the $\tilde{B}$ function becomes negative. In these cases, the radiative event generated with POWHEG starting from the underlying-Born configuration $\overline{\boldsymbol{\Phi}}_{2}$ is negative-weighted. We have checked that this occurrence is rare, so that the benefits of having positive-weighted events are not spoilt. Moreover, in the POWHEG-BOX package, a procedure (called "folded" integration) to reduce further the occurrence of these events is available, as explained in Sect. 4.1 of [51]. By using it, we have verified that the occurrence of negative-weighted events can be further reduced also for the $W t$-channel case. This also implies that the function $\bar{B}$ is positive, as in the other POWHEG implementations.

Quantitatively, in the event sample we generated to produce the plots shown in Sect. 3, the fraction of negative-weighted events was 0.05 . By using the aforementioned folded integration, this fraction can be reduced down to 0.005 , becoming therefore completely negligible.

- For 3-body kinematic configurations where $\mathcal{R}^{\mathrm{DS}}$ is not positive, another problem can occur during the generation of the hardest radiation. We recall that, to generate the hardest radiation, the POWHEG algorithm works by finding an upper-bound for the ratio $\mathcal{R} / \mathcal{B}$, assuming that this ratio is always positive. Negative values can therefore spoil the accuracy of the method. We have checked that this happens with a certain frequency only close to the doubly resonant region. Since this is the kinematic region where the separation of $t \bar{t}$ and $W t$ is already particularly critical, we decided to explicitly avoid to generate radiative events when close to the doubly resonant peak. This has been obtained using a theta function that vanishes in this region, i.e. in DS the following substitution is performed when $\mathcal{R} / \mathcal{B}$ is evaluated:

$$
\mathcal{R}^{\mathrm{DS}} \rightarrow \mathcal{R}^{\mathrm{DS}} \times \theta\left(\left|m_{W \bar{b}}-m_{t}\right|-\kappa \Gamma_{t}\right) .
$$

We have tested the effects of this cutoff trying values of order 1 for $\kappa$, in order to avoid introducing effects from this parameter in phase-space regions where it is not needed. The outcome of this check is that, at the end of the event generation (i.e. after the shower and the hadronization stage), no problems caused by this cutoff are present, since results depend negligibly on the value of $\kappa$. The only observable where some dependence was observed is the differential distribution of $m_{W \bar{b}}$ close to the doubly resonant peak.

Results for this observable obtained using different values for $\kappa$ are reported in Fig. 3. Curves are obtained after showering POWHEG samples with PYTHIA, and $m_{W \bar{b}}$ was computed from the momenta of the primary $W$-boson (the $W$-boson produced in the hard process) and the hardest $\bar{b}$-flavoured hadron in the event. The effect of different values of the cutoff can be seen in the left-hand plot: the region affected is the one close to the top-mass peak, as expected, and some residual dependence can be also observed in the region $150 \mathrm{GeV} \lesssim m_{W \bar{b}}<m_{t}$. As already stated, this is the region where the definition of the $W t$-channel is not meaningful. However, in order to check whether the introduction of a cutoff introduces unphysical behavior only in the doubly resonant region, where $W t$-channel production is intrinsically ill defined, we studied the same distribution in presence of a set of analysis cuts designed to isolate the $W t$-signal. These "Wt-signal cuts" are reported explicitly in Sect. 3. As expected, from the panel on the right of Fig. 3 we see that

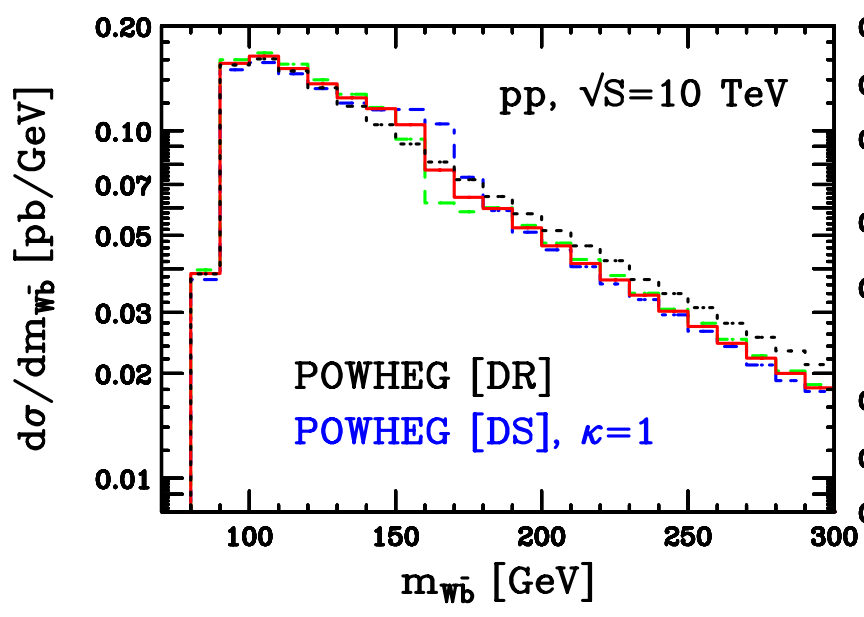

Fig. 3 Comparison between POWHEG (interfaced to PYTHIA) results for the variable $m_{W \bar{b}}$ at the LHC $p p$ collider $(\sqrt{S}=10 \mathrm{TeV})$, obtained using different values for the cutoff $\kappa$ in the DS case (DS, $\kappa=1$ : blue dotdashes-DS, $\kappa=3$ : red solid-DS, $\kappa=5$ : green dashes-DR:

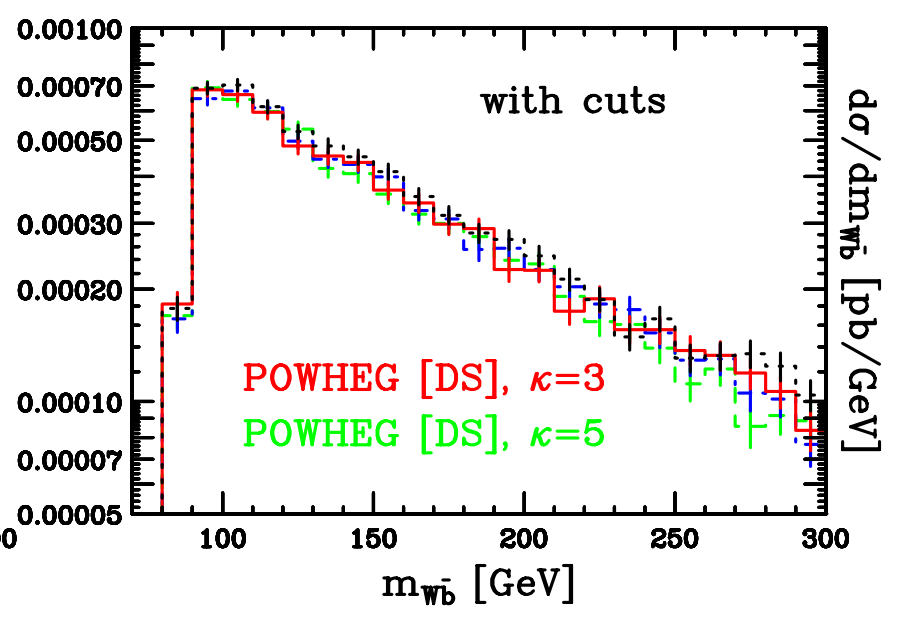

black dots). Curves in the left panel are obtained without cuts, those in the right panel are obtained with the " $W t$-signal cuts" described at the end of Sect. 3. Uncertainties indicated by the vertical bars in the right panel are only statistical 

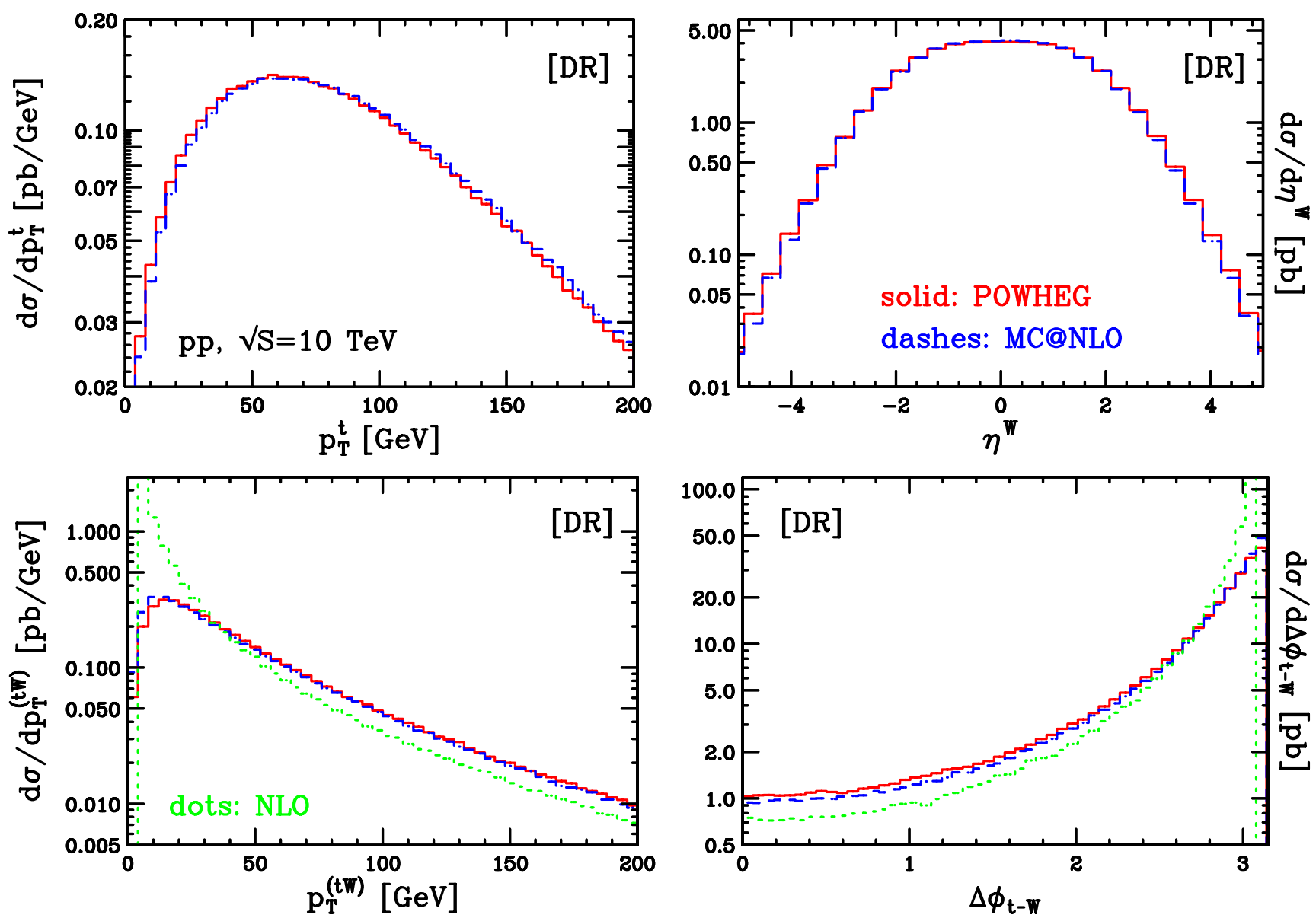

Fig. 4 Comparisons between POWHEG (interfaced to HERWIG) and MC@NLO results at the LHC $p p$ collider $(\sqrt{S}=10 \mathrm{TeV})$, obtained with the DR prescription. NLO results are also shown in the lower panel

when signal cuts are in place, the dependence of the DS result from the cutoff becomes negligible, and moreover the agreement between the prediction from DR and DS is very good. Further evidence of this agreement will be shown for other observables in plots at the end of Sect. 3.

Although we are aware that the introduction of a cutoff may be considered unappealing, we found it a reasonable choice to handle with the problem of having negative values for $\mathcal{R} / \mathcal{B}$ in the POWHEG framework: visible effects were observed only in one specific observable, in the region where the calculation is, however, unreliable, and in absence of reasonable cuts. When cuts are in place, cutoff dependence turns out to be negligible also for this observable.

We also recall that the presence of this cutoff in our implementation does not affect the issue of negative weights, since $\kappa$ is used only in the generation of the hardest radiation. The results shown in Sect. 3 have been obtained with $\kappa=3$.

We used MadGraph to calculate the term $\mathcal{C}^{\text {SUB }}$. We also notice that to avoid the divergence of the internal top-quark propagator when $\left(k_{1}+k_{3}\right)^{2} \rightarrow m_{t}^{2}$, in DS a non-vanishing value for the top width is needed. ${ }^{5}$

\section{Results}

In this section we present our results obtained after showering with HERWIG 6.510 and PYTHIA 6.4.22 the partonic events generated with POWHEG. We considered top production at the LHC, with an hadronic center-of-mass energy $\sqrt{S}=10 \mathrm{TeV}$. All results have been obtained assuming that the top-quark decays semileptonically $(t \rightarrow b \bar{\ell} \nu)$ and that the $W$-boson involved in the hard scattering decays leptonically $\left(W^{-} \rightarrow \ell \bar{v}\right)$. Branching ratios have been removed, so that plots are normalized to the total cross section.

We have used the CTEQ6M [62] set for the parton distribution functions and the associated value of $\Lambda_{\overline{\mathrm{MS}}}^{(5)}=$ $0.226 \mathrm{GeV}$. Furthermore, as discussed in [23, 32], we use

\footnotetext{
${ }^{5}$ We recall that to obtain the proper cancellation of soft and collinear divergences, $\mathcal{M}^{W t}$ has to be calculated with $\Gamma_{t}=0$.
} 

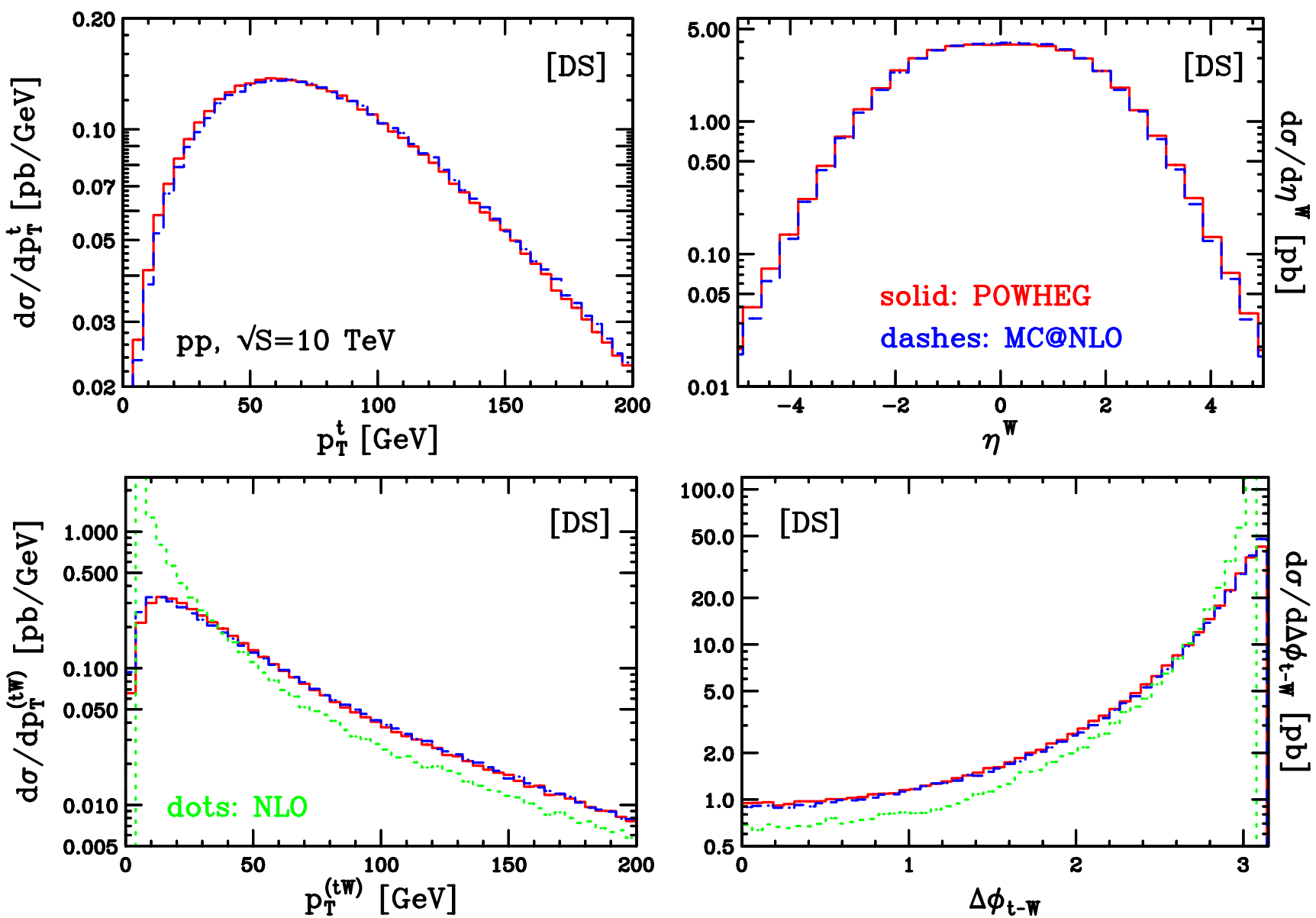

Fig. 5 Comparisons between POWHEG (interfaced to HERWIG) and MC@NLO results at the LHC $p p$ collider $(\sqrt{S}=10 \mathrm{TeV})$, obtained with the DS prescription. NLO results are also shown in the lower panel

a rescaled value $\Lambda_{\mathrm{MC}}=1.569 \Lambda_{\mathrm{MS}}^{(5)}$ in the expression for $\alpha_{\mathrm{S}}$ appearing in the Sudakov form factors, in order to achieve next-to-leading logarithmic accuracy.

Although the matrix-element calculation has been performed in the massless-quark limit, the lower cutoff in the generation of the radiation has been fixed according to the mass of the emitting quark. The lower bound on the transverse momentum for the emission off a massless emitter $(u, d, s)$ has been set to the value $p_{\mathrm{T}}^{\min }=\sqrt{5} \Lambda_{\mathrm{MC}}$. We instead choose $p_{\mathrm{T}}^{\min }$ equal to $m_{c}$ or $m_{b}$ when the gluon is emitted by a charm or a bottom quark, respectively. We set $m_{c}=1.55 \mathrm{GeV}$ and $m_{b}=4.95 \mathrm{GeV}$.

The renormalization and factorization scales have been taken equal to the transverse momentum of the radiated light parton during the generation of radiation, in accordance with the POWHEG formalism. We have also taken into account properly the heavy-flavor thresholds in the running of $\alpha_{\mathrm{S}}$ and in the PDF's, by changing the number of active flavors when the renormalization or factorization scales cross a mass threshold. In the $\bar{B}$ calculation, instead, $\mu_{R}$ and $\mu_{F}$ have been chosen equal to the top-quark mass, whose value has been fixed to $m_{t}=175 \mathrm{GeV}$. In the DS approach, the amplitudes where doubly resonant graphs are present and the subtraction term $\mathcal{C}^{\mathrm{SUB}}$ have been calculated with $\Gamma_{t}=1.7 \mathrm{GeV}$.

To assess the validity of the approximations and the choices we made, we compare our results (obtained both with DR and DS) with the MC@NLO outputs.

In Fig. 4 we show a comparison between POWHEG and MC@NLO, obtained with the DR prescription, without applying any cut on the final-state particles. POWHEG results have been obtained using the HERWIG parton shower, in order to minimize differences arising from different shower algorithms and hadronization models. In the upper panel of Fig. 4 we show the transverse momentum of the top quark $\left(p_{\mathrm{T}}^{t}\right)$ and the pseudorapidity of the $W$-boson $\left(\eta^{W}\right)$ produced in the hard process (i.e. not the $W$-boson present in the decay chain of the top quark). As expected, we found very good agreement between the two results, since the shape of these two distributions is due mainly to the fixed-order result. In the lower panel, we show instead $p_{\mathrm{T}}^{(t W)}$, the transverse momentum of the system made by the top quark and 

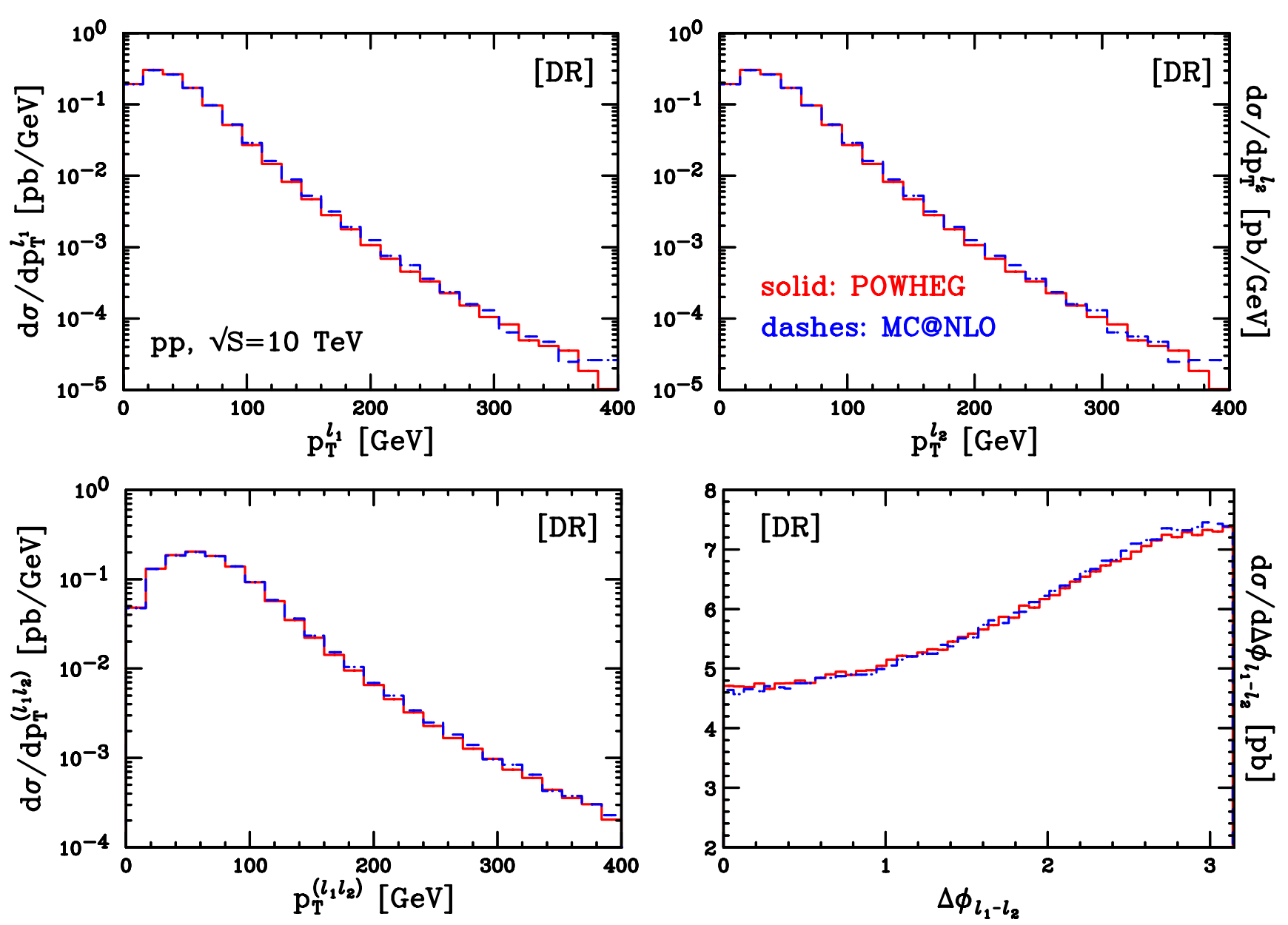

Fig. 6 Comparisons between POWHEG (interfaced to HERWIG) and MC@NLO results at the LHC pp collider $(\sqrt{S}=10 \mathrm{TeV})$, obtained with the DR prescription, for leptonic quantities

the $W$ boson, and $\Delta \phi_{t-W}$, the difference between the azimuthal angles of the two particles. These two quantities are significant because their shape is affected by Sudakov suppression effects due to the resummation performed by parton showers. To stress the size of these effects, we also superimposed the fixed-order (NLO) prediction to the last two plots. We observe good agreement between showered results, and the expected difference with the NLO curve, where the cancellation of soft and collinear divergences takes place only at the edge of the distributions (at $p_{\mathrm{T}}^{(t W)}=0$ and $\Delta \phi_{t-W}=\pi$, respectively).

In Fig. 5 we show the same set of plots, obtained with the DS prescription. The same considerations made above are valid also for this case. We recall that the plots shown in Figs. 4 and 5 have been obtained using the top-quark and the $W$-boson momenta extracted from the parton shower history. Therefore, these quantities are not measurable in a real detector. Nevertheless, since they are useful to check the implementation, we have shown the corresponding results.

We have also included the generation of the top-quark and the $W$-boson decay products, according to the method originally proposed in [63]. This enables the generation of events in which spin-correlation effects in the productiondecay stage are taken into account with leading-order accuracy. In Figs. 6 and 7 we show a comparison between MC@NLO and POWHEG for some relevant leptonic distributions. We plot the transverse momenta of the hardest $\left(p_{\mathrm{T}}^{\ell_{1}}\right)$ and the second-hardest $\left(p_{\mathrm{T}}^{\ell_{2}}\right)$ charged lepton in the event. We also show $p_{\mathrm{T}}^{\left(\ell_{1} \ell_{2}\right)}$, the transverse momentum of the system made by $\ell_{1}$ and $\ell_{2}$, and $\Delta \phi_{\ell_{1}-\ell_{2}}$, the difference between the azimuthal angles of the two leptons. Here, again, we found very good agreement in both the DR and the DS case. We recall that a quantity like $\Delta \phi_{\ell_{1}-\ell_{2}}$ is sensitive to spincorrelation effects, as we have verified by running the code with the decay-generation procedure switched off and letting the shower perform isotropic decays.

In [15] the use of a $b$-veto as a method to discriminate between the $W t$ and the $t \bar{t}$ processes was proposed. This idea was also reconsidered in [36], where its applicability was studied in the context of a NLO result merged with a parton shower. We have performed a similar exercise using the results obtained with POWHEG and showered with HERWIG. 

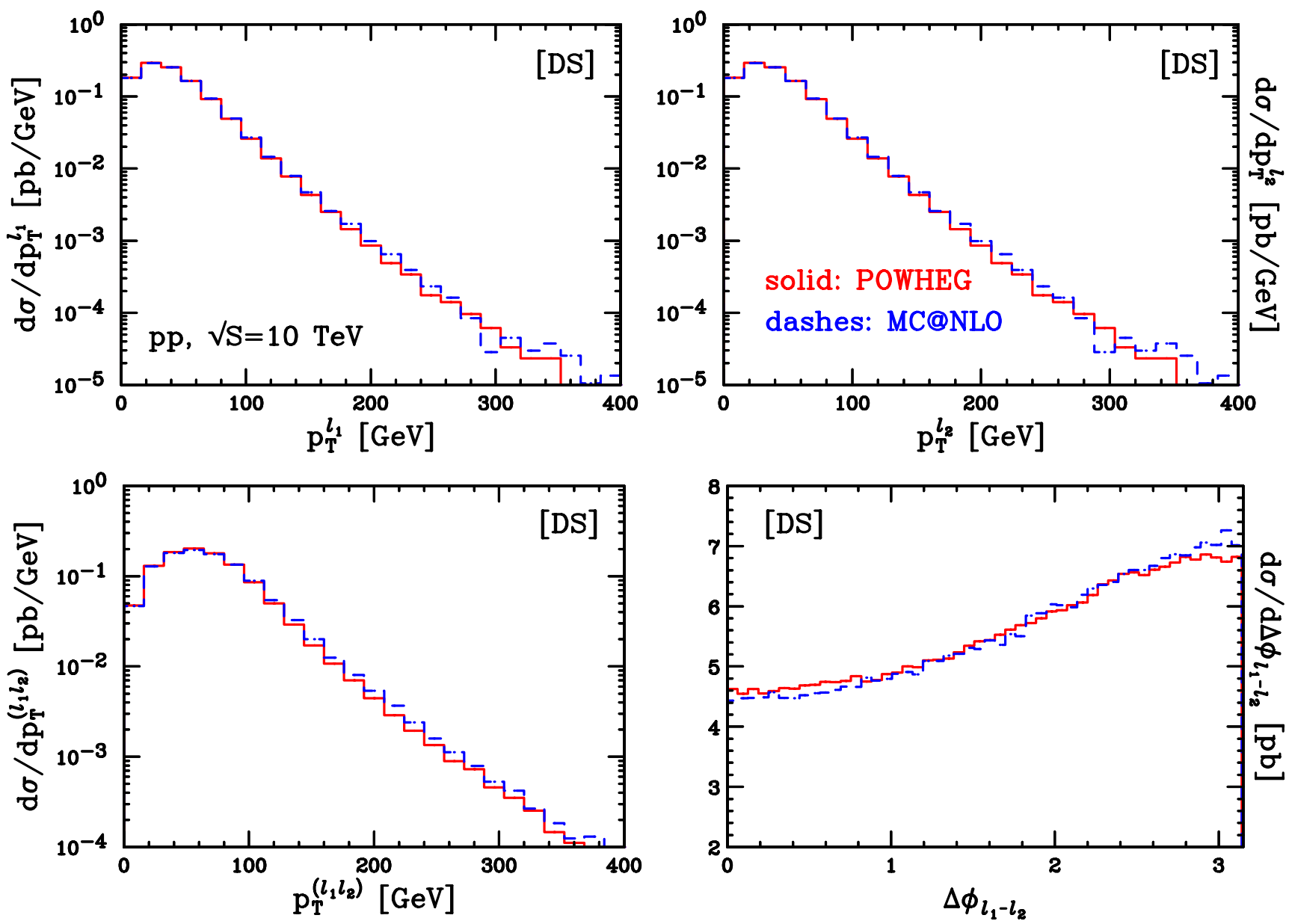

Fig. 7 Comparisons between POWHEG (interfaced to HERWIG) and MC@NLO results at the LHC $p p$ collider $(\sqrt{S}=10 \mathrm{TeV})$, obtained with the DS prescription, for leptonic quantities

The $b$-veto condition is defined as follows: after having sorted in decreasing $p_{\mathrm{T}}$ all the $b$-flavoured hadrons present in the final state, an event is kept if the second-hardest $b$-flavoured hadron among those with central pseudorapidity $\left(\eta^{B} \leq 2.5\right)$ has $p_{\mathrm{T}}^{B}<p_{\mathrm{T}}^{(b-v e t o)}$, otherwise it is discarded. ${ }^{6}$ In our analysis procedure we chose $p_{\mathrm{T}}^{(b \text {-veto })}=50 \mathrm{GeV}$.

As expected, we observe that the difference between DR and DS results is reduced when the $b$-veto is applied: in fact, although we have not performed a detailed study including also uncertainties due to scale variations, we found that the ratios between the total NLO cross sections using DR and DS are as follows:

$\frac{\sigma_{(\mathrm{DS})}}{\sigma_{(\mathrm{DR})}}=0.95, \quad \frac{\sigma_{(\mathrm{DS})}^{(b-\text { veto })}}{\sigma_{(\mathrm{DR})}^{(b-\mathrm{veto})}}=0.98$.

${ }^{6}$ If all the $b$-hadrons in the central pseudorapidity region have $p_{\mathrm{T}}^{B}<$ $p_{\mathrm{T}}^{(b \text {-veto })}$, or if the second-hardest $b$-hadron has a large pseudorapidity, the event is kept.
As the MC@NLO authors already pointed out, despite the fact that the $b$-veto reduces the difference between DR and DS total cross sections, it is not guaranteed that all the differential distributions are affected in the same way. To address this question, in Fig. 8 we show the effect of the $b$-veto cut for two transverse-momentum spectra. In the upper panel we plot the results for $p_{\mathrm{T}}^{(t W)}$, obtained with the DR and the DS procedures, before and after imposing the $b$-veto. The curves in the upper-left panel are obtained without cuts, those on the upper-right have been obtained keeping only the events that fulfill the veto condition. It can be seen that the mismatch between DR and DS in the high- $p_{\mathrm{T}}$ tail is less sizeable when the $b$-veto is applied, which is the expected result. Since in [36] it was noticed that the transverse momentum of the system made by the two hardest leptons turns out to be sensitive to the treatment of the doubly resonant region, in the lower panel of Fig. 8 we show also the predictions for $p_{\mathrm{T}}^{\left(\ell_{1} \ell_{2}\right)}$. Also for this quantity the effect of the $b$-veto is to reduce the differences between DR and DS, as the plot in the lower-right panel shows, in accordance with what has been found in [36]. 

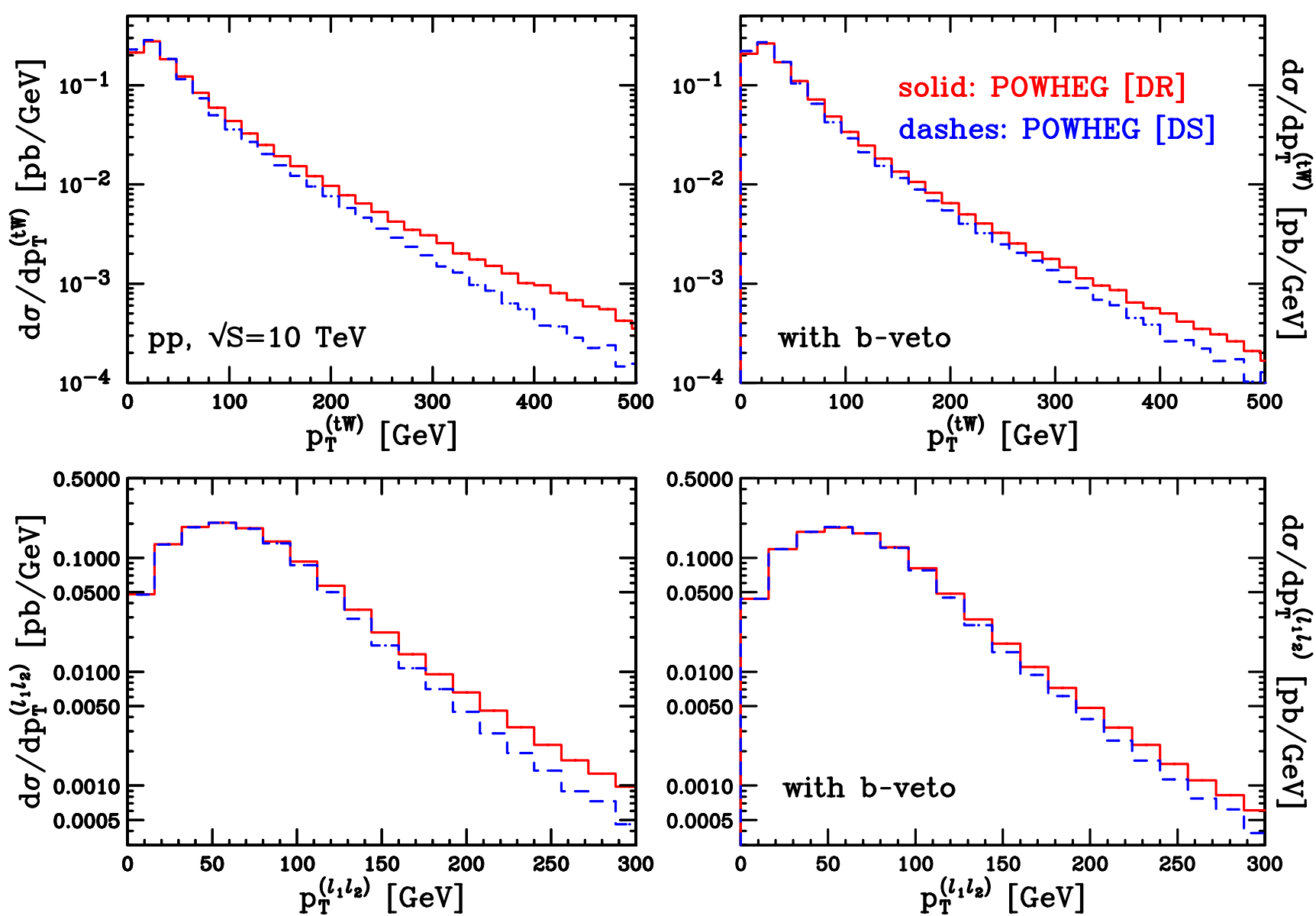

Fig. 8 Comparisons between POWHEG (interfaced to HERWIG) results obtained with the DR and DS prescriptions. Plots in the right panel have been obtained with the $b$-veto described in the text

The conclusions of this analysis can be summarized as follows. We checked that the $b$-veto reduces the differences between DR and DS. This is the expected result, since the $b$-veto was originally proposed by the authors of [15] to reduce interference effects from $t \bar{t}$ production. In fact, by requiring a $b$-veto, one reduces the number of events with two hard and central $b$-flavoured hadrons, which is indeed one of the typical signatures of $t \bar{t}$ production. The fact that DR and DS total cross sections become closer when the $b$-veto is applied is also in accordance with the interpretation of the difference between DR and DS being a measure of the interference between $W t$ and $t \bar{t}$ production. This interpretation is supported by the plots in Fig. 8, where it is shown that the difference between DR and DS is reduced also for differential distributions, when the $b$-veto is applied. However, the plots in Fig. 8 show also that observables are potentially affected by the $b$-veto non uniformly (i.e. ratios between DR and DS results can be bindependent). This suggests that particular care should be taken when one performs a full analysis where the contribution from the $W t$-channel process is supposed to be rele- vant, since the size of interference effects may depend on the cuts applied. As was already observed by the MC@NLO authors, a comparison between DR and DS predictions gives an estimate of the theoretical uncertainty due to these effects.

We have also performed some comparisons between DR and DS results obtained with the PYTHIA shower. In order to maximize consistency with the POWHEG prescriptions, we used the $p_{\mathrm{T}}$-ordered shower. In Fig. 9 we show the pseudorapidity and the transverse momentum of the system made by the two hardest leptons, while in Fig. 10 the transverse momentum of the hardest non $b$-flavoured jet $\left(p_{\mathrm{T}}^{j_{1}}\right)$ and for the hardest jet that contains a $b$-flavoured hadron $\left(p_{\mathrm{T}}^{b j_{1}}\right)$ are shown. The plots on the left have been obtained without imposing any cut. Instead, the plots on the right have been obtained using cuts similar to the " $W t$-signal cuts" of [54]. Jets have been defined according to the $k_{\mathrm{T}}$ algorithm [64], as implemented in the FASTUET package [65], setting $R=0.7$ and imposing a lower $10 \mathrm{GeV}$ cut on jet transverse momenta. To accept an event, we required the following properties: 

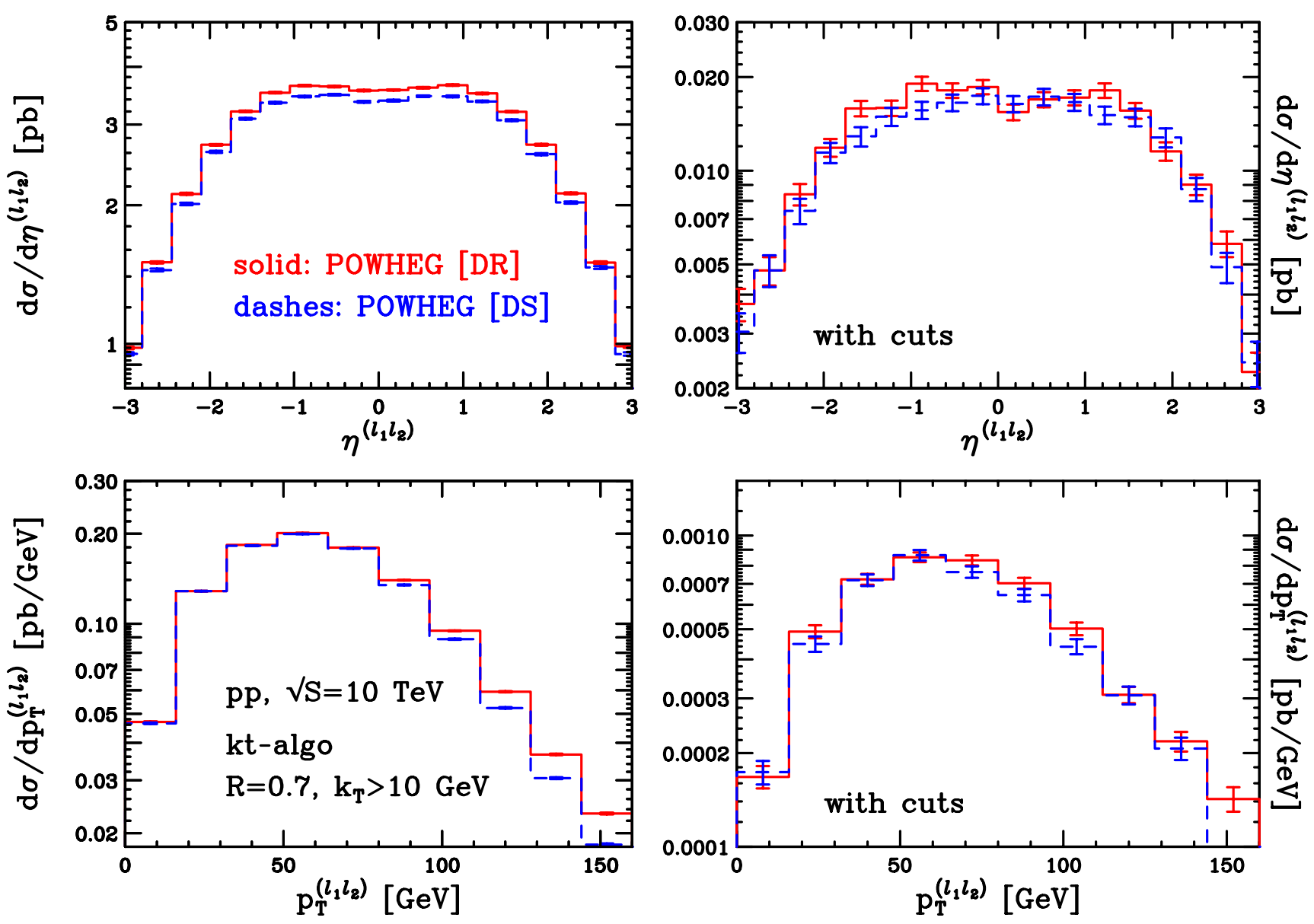

Fig. 9 Comparisons between POWHEG (interfaced to PYTHIA) results obtained with the DR and DS prescriptions, for leptonic quantities. Plots in the right panel have been obtained with the " $W t$-cuts" described in the text. Uncertainties indicated by the vertical bars are only statistical

- There is exactly one $b$-jet with $p_{\mathrm{T}}^{j}>50 \mathrm{GeV}$ and $\left|\eta^{j}\right|<$ 2.5. A $b$-jet is defined as a jet that contains at least one $b$-flavoured hadron and has $p_{\mathrm{T}}^{j}>25 \mathrm{GeV}$ and $\left|\eta^{j}\right|<2.5$.

- There are at least two light-flavoured jets with $p_{\mathrm{T}}^{j}>$ $25 \mathrm{GeV}$ and $\left|\eta^{j}\right|<2.5$. The invariant mass of the system made by the two hardest jets among these light-flavoured jets has to lie within 55 and $85 \mathrm{GeV}$.

- There is one lepton with $p_{\mathrm{T}}^{\ell}>25 \mathrm{GeV}$ and $\left|\eta^{\ell}\right|<2.5$. This lepton has also to be isolated with respect to the $b$-jet and the two light-flavoured jets, i.e. its distance from the jets in the $(\eta, \phi)$ plane has to be larger than 0.4.

- The missing transverse energy is larger than $25 \mathrm{GeV}$.

Although we have not performed as detailed a study as the one of [54], from Figs. 9 and 10 we observe that the DR and the DS predictions are consistent (within the statistical accuracy) also when the above cuts are applied, as was observed in the aforementioned work.

\section{Conclusions}

In this paper we have described the implementation of $W t$-channel single-top production at next-to-leading order in $\mathrm{QCD}$, in the POWHEG framework. We have used the POWHEG-BOX package, which is a program that automates the algorithm first proposed in [22] and then carefully described in [23]. Having used this package, we described how we calculated the main needed ingredients.

Since NLO corrections to single-top production in the $W t$-channel are known not to be well defined (real contributions interfere with $t \bar{t}$ production), we decided to follow the same strategy originally proposed by the MC@NLO authors in [36]: we included two definitions of the NLO corrections, known as DR (Diagram Removal) and DS (Diagram Subtraction), both of which can be used when the merging of the fixed-order result with parton showers is performed. Moreover, the difference of results obtained with these two prescriptions gives an estimate of the size of interference effects. We have described how we included the two prescriptions in POWHEG, and how we dealt with DS and the problem of its exact implementation within the POWHEG method.

To check the correctness of the whole implementation and to assess the validity of the choices we made, results have been compared with the MC@NLO program for the LHC, where $W t$ production is relevant: we found very good 

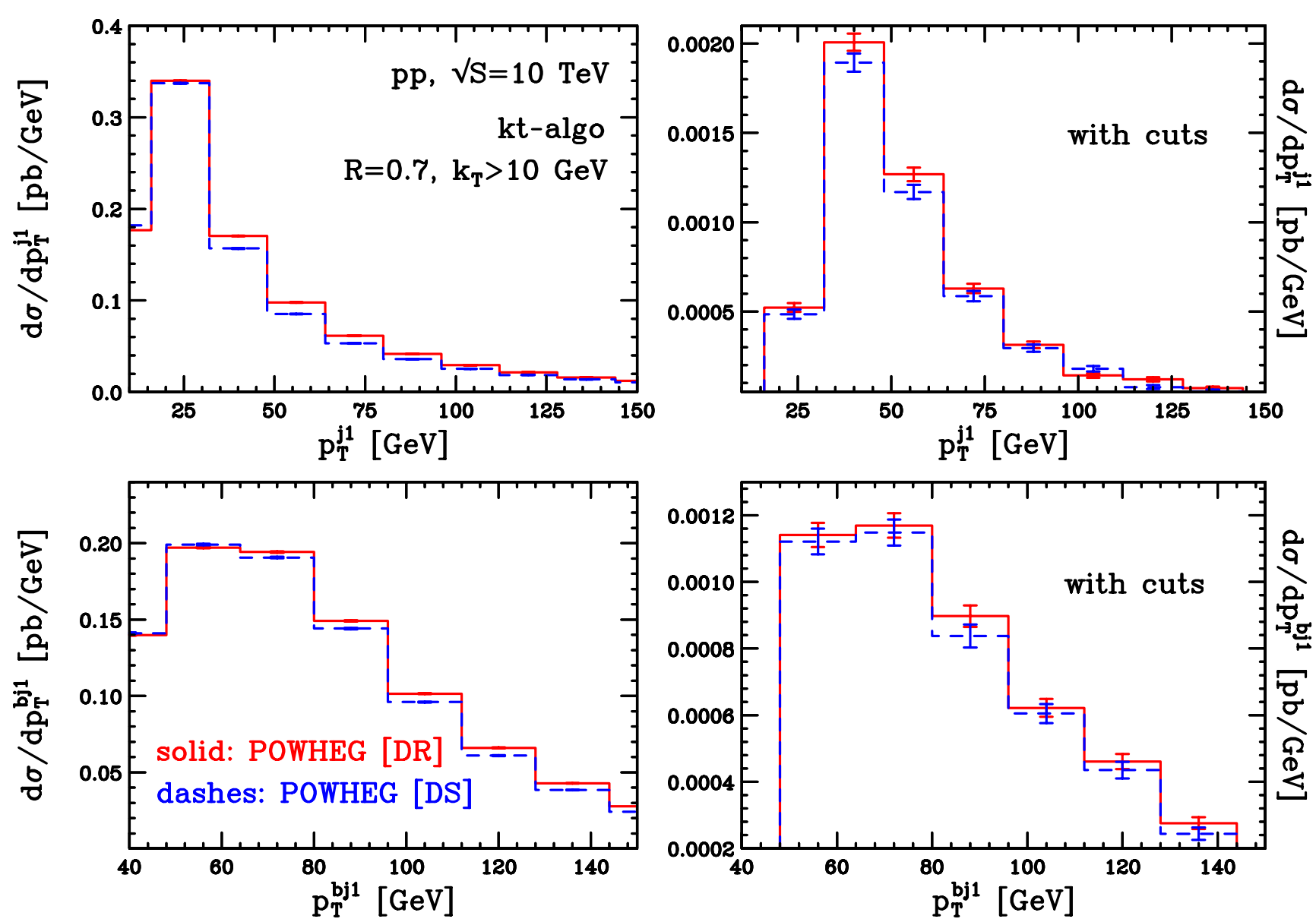

Fig. 10 Comparisons between POWHEG (interfaced to PYTHIA) results obtained with the DR and DS prescriptions. Plots in the right panel have been obtained with the "Wt-cuts" described in the text. Uncertainties indicated by the vertical bars are only statistical

agreement, both for DR and DS. We have also compared DR and DS results when a $b$-veto is imposed. We found that a $b$-veto reduces interference effects with $t \bar{t}$, the difference between DR and DS results becoming smaller when the veto is in place. Moreover, we have also presented some results obtained with the PYTHIA shower and with typical defining cuts for the $W t$ signal. Although we have not performed as full an analysis as the one reported in [54], good agreement between DR and DS has been found also when typical $W t$-channel cuts are applied.

The main purpose of this work was the completion of the work presented in [41], where the POWHEG implementation of single-top $s$ - and $t$-channel was described. Therefore, at present all single-top processes can be simulated in the context of a NLO+PS approach with POWHEG as well as with MC@NLO. We also stress that, in POWHEG, $W t$-channel was the missing process among the ones relevant for $H \rightarrow W W$ searches: Higgs production via gluon [37] and vector-boson fusion [42] are already available, as well as $t \bar{t}$ [33] and $V V$ [66] production, which (together with single-top $W t$ ) are the main backgrounds.
The computer code for this POWHEG implementation will soon be available within the public branch of the POWHEGBOX package, and it can be downloaded at the site

$$
\text { http://virgilio.mib.infn.it/ nason/POWHEG }
$$

Acknowledgements I would like to particularly thank C. White, for providing me with the one-loop corrections for this process, for many useful discussions on this project, and also for comments on the manuscript. I would also like to thank R. Frederix and G. Zanderighi for helpful hints on the usage of MadGraph and QCDloop. I am also grateful to S. Alioli and K. Hamilton for comments on the manuscript.

Open Access This article is distributed under the terms of the Creative Commons Attribution Noncommercial License which permits any noncommercial use, distribution, and reproduction in any medium, provided the original author(s) and source are credited.

\section{References}

1. T. Aaltonen et al. (CDF Collaboration), First observation of electroweak single top quark production. Phys. Rev. Lett. 103, 092002 (2009). arXiv:0903.0885 [hep-ex] 
2. V.M. Abazov et al. (D0 Collaboration), Observation of single top-quark production. Phys. Rev. Lett. 103, 092001 (2009). arXiv:0903.0850 [hep-ex]

3. J. Alwall et al., Is $V(t b)=1$ ? Eur. Phys. J. C 49, 791 (2007). arXiv:hep-ph/0607115

4. G. Mahlon, S.J. Parke, Improved spin basis for angular correlation studies in single top quark production at the Tevatron. Phys. Rev. D 55, 7249 (1997). arXiv:hep-ph/9611367

5. G. Mahlon, S.J. Parke, Single top quark production at the LHC: Understanding spin. Phys. Lett. B 476, 323 (2000). arXiv:hep-ph/9912458

6. T.M.P. Tait, C.P.P. Yuan, Single top quark production as a window to physics beyond the standard model. Phys. Rev. D 63, 014018 (2001). arXiv:hep-ph/0007298

7. Q.H.P. Cao, J. Wudka, C.P. Yuan, Search for New Physics via Single Top Production at the LHC. Phys. Lett. B 658, 50 (2007). arXiv:0704.2809 [hep-ph]

8. M. Dittmar, H.K. Dreiner, How to find a Higgs boson with a mass between $155-\mathrm{GeV}-180-\mathrm{GeV}$ at the LHC. Phys. Rev. D 55, 167 (1997). arXiv:hep-ph/9608317

9. G. Bordes, B. van Eijk, Calculating QCD corrections to single top production in hadronic interactions. Nucl. Phys. B 435, 23 (1995)

10. W.T. Giele, S. Keller, E. Laenen, QCD corrections to $W$ boson plus heavy quark production at the Tevatron. Phys. Lett. B 372, 141 (1996). arXiv:hep-ph/9511449

11. T. Stelzer, Z. Sullivan, S. Willenbrock, Single top quark production via $W$-gluon fusion at next-to-leading order. Phys. Rev. D 56, 5919 (1997). arXiv:hep-ph/9705398

12. B.W. Harris, E. Laenen, L. Phaf, Z. Sullivan, S. Weinzierl, The Fully differential single top quark cross-section in next to leading order QCD. Phys. Rev. D 66, 054024 (2002). arXiv:hep-ph/0207055

13. S. Zhu, Next-to-leading order QCD corrections to $\mathrm{b} g \rightarrow \mathrm{t} \mathrm{W}$-at the CERN large hadron collider. Phys. Lett. B 524, 283 (2002). Erratum Phys. Lett. B 537, 351 (2002)

14. J.M. Campbell, R.K. Ellis, F. Tramontano, Single top production and decay at next-to-leading order. Phys. Rev. D 70, 094012 (2004). arXiv:hep-ph/0408158

15. J.M. Campbell, F. Tramontano, Next-to-leading order corrections to $\mathrm{W}$ t production and decay. Nucl. Phys. B 726, 109 (2005). arXiv:hep-ph/0506289

16. Q.H. Cao, R. Schwienhorst, C.P. Yuan, Next-to-leading order corrections to single top quark production and decay at Tevatron. 1. $s^{-}$channel process. Phys. Rev. D 71, 054023 (2005). arXiv:hep-ph/0409040

17. Q.H. Cao, R. Schwienhorst, J.A. Benitez, R. Brock, C.P. Yuan, Next-to-leading order corrections to single top quark production and decay at the Tevatron: $2 . t^{-}$channel process. Phys. Rev. D 72, 094027 (2005). arXiv:hep-ph/0504230

18. N. Kidonakis, Single top production at the Tevatron: Threshold resummation and finite-order soft gluon corrections. Phys. Rev. D 74, 114012 (2006). arXiv:hep-ph/0609287

19. J.M. Campbell, R. Frederix, F. Maltoni, F. Tramontano, Nextto-leading-order predictions for t-channel single-top production at hadron colliders. Phys. Rev. Lett. 102, 182003 (2009). arXiv:0903.0005 [hep-ph]

20. J.M. Campbell, R. Frederix, F. Maltoni, F. Tramontano, NLO predictions for t-channel production of single top and fourth generation quarks at hadron colliders. J. High Energy Phys. 0910, 042 (2009). arXiv:0907.3933 [hep-ph]

21. S. Frixione, B.R. Webber, Matching NLO QCD computations and parton shower simulations. J. High Energy Phys. 0206, 029 (2002). arXiv:hep-ph/0204244

22. P. Nason, A new method for combining NLO QCD with shower Monte Carlo algorithms. J. High Energy Phys. 0411, 040 (2004). arXiv:hep-ph/0409146
23. S. Frixione, P. Nason, C. Oleari, Matching NLO QCD computations with Parton Shower simulations: the POWHEG method. J. High Energy Phys. 0711, 070 (2007). arXiv:0709.2092 [hep-ph]

24. Z. Nagy, D.E. Soper, Matching parton showers to NLO computations. J. High Energy Phys. 0510, 024 (2005). arXiv:hep-ph/0503053

25. W.T. Giele, D.A. Kosower, P.Z. Skands, A simple shower and matching algorithm. Phys. Rev. D 78, 014026 (2008). arXiv:0707.3652 [hep-ph]

26. N. Lavesson, L. Lonnblad, Extending CKKW-merging to oneloop matrix elements. J. High Energy Phys. 0812, 070 (2008). arXiv:0811.2912 [hep-ph]

27. O. Latunde-Dada, S. Gieseke, B. Webber, A positive-weight nextto-leading-order Monte Carlo for $\mathrm{e}^{+} \mathrm{e}^{-}$annihilation to hadrons. J. High Energy Phys. 0702, 051 (2007). arXiv:hep-ph/0612281

28. O. Latunde-Dada, Herwig Monte Carlo at next-to-leading order for $e^{+} e^{-}$annihilation and lepton pair production. J. High Energy Phys. 0711, 040 (2007). arXiv:0708.4390 [hep-ph]

29. O. Latunde-Dada, Applying the POWHEG method to top pair production and decays at the ILC. Eur. Phys. J. C 58, 543 (2008). arXiv:0806.4560 [hep-ph]

30. S. Frixione, P. Nason, B.R. Webber, Matching NLO QCD and parton showers in heavy flavour production. J. High Energy Phys. 0308, 007 (2003). arXiv:hep-ph/0305252

31. S. Frixione, E. Laenen, P. Motylinski, B.R. Webber, Single-top production in MC@ NLO. J. High Energy Phys. 0603, 092 (2006). arXiv:hep-ph/0512250

32. P. Nason, G. Ridolfi, A positive-weight next-to-leading-order Monte Carlo for $\mathrm{Z}$ pair hadroproduction. J. High Energy Phys. 0608, 077 (2006). arXiv:hep-ph/0606275

33. S. Frixione, P. Nason, G. Ridolfi, A positive-weight next-toleading-order Monte Carlo for heavy flavour hadroproduction. J. High Energy Phys. 0709, 126 (2007). arXiv:0707.3088 [hep-ph]

34. S. Alioli, P. Nason, C. Oleari, E. Re, NLO vector-boson production matched with shower in POWHEG. J. High Energy Phys. 0807, 060 (2008). arXiv:0805.4802 [hep-ph]

35. K. Hamilton, P. Richardson, J. Tully, A positive-weight nextto-leading order Monte Carlo simulation of Drell-Yan vector boson production. J. High Energy Phys. 0810, 015 (2008). arXiv:0806.0290 [hep-ph]

36. S. Frixione, E. Laenen, P. Motylinski, B.R. Webber, C.D. White, Single-top hadroproduction in association with a W boson. J. High Energy Phys. 0807, 029 (2008). arXiv:0805.3067 [hep-ph]

37. S. Alioli, P. Nason, C. Oleari, E. Re, NLO Higgs boson production via gluon fusion matched with shower in POWHEG. J. High Energy Phys. 0904, 002 (2009). arXiv:0812.0578 [hep-ph]

38. K. Hamilton, P. Richardson, J. Tully, A positive-weight next-toleading order Monte Carlo simulation for Higgs boson production. J. High Energy Phys. 0904, 116 (2009). arXiv:0903.4345 [hep$\mathrm{ph}]$

39. A. Papaefstathiou, O. Latunde-Dada, NLO production of $W$ ' bosons at hadron colliders using the MC@NLO and POWHEG methods. J. High Energy Phys. 0907, 044 (2009). arXiv:0901.3685 [hep-ph]

40. O. Latunde-Dada, MC@NLO for the hadronic decay of Higgs bosons in associated production with vector bosons. J. High Energy Phys. 0905, 112 (2009). arXiv:0903.4135 [hep-ph]

41. S. Alioli, P. Nason, C. Oleari, E. Re, NLO single-top production matched with shower in POWHEG: s- and t-channel contributions. J. High Energy Phys. 0909, 111 (2009). Erratum J. High Energy Phys. 1002, 011 (2010). arXiv:0907.4076 [hep-ph]

42. P. Nason, C. Oleari, NLO Higgs boson production via vectorboson fusion matched with shower in POWHEG. J. High Energy Phys. 1002, 037 (2010). arXiv:0911.5299 [hep-ph]

43. C. Weydert et al., Charged Higgs boson production in association with a top quark in MC@ NLO. Eur. Phys. J. C 67, 617 (2010). arXiv:0912.3430 [hep-ph] 
44. P. Torrielli, S. Frixione, Matching NLO QCD computations with PYTHIA using MC@NLO. J. High Energy Phys. 1004, 110 (2010). arXiv:1002.4293 [hep-ph]

45. G. Corcella et al., HERWIG 6.5: an event generator for hadron emission reactions with interfering gluons (including supersymmetric processes). J. High Energy Phys. 0101, 010 (2001). arXiv:hep-ph/0011363

46. T. Sjostrand, S. Mrenna, P.Z. Skands, PYTHIA 6.4 physics and manual. J. High Energy Phys. 0605, 026 (2006). arXiv:hep-ph/0603175

47. M. Bahr et al., Herwig++ physics and manual. Eur. Phys. J. C 58, 639 (2008). arXiv:0803.0883 [hep-ph]

48. J.M. Butterworth et al., The tools and Monte Carlo working group summary report. arXiv:1003.1643 [hep-ph]

49. S. Hoeche, F. Krauss, M. Schonherr, F. Siegert, Automating the POWHEG method in Sherpa. arXiv:1008.5399 [hep-ph]

50. P. Nason, Recent developments in POWHEG. PoS RADCOR2009, 018 (2010). arXiv:1001.2747 [hep-ph]

51. S. Alioli, P. Nason, C. Oleari, E. Re, A general framework for implementing NLO calculations in shower Monte Carlo programs: the POWHEG BOX. J. High Energy Phys. 1006, 043 (2010). arXiv:1002.2581 [hep-ph]

52. A.S. Belyaev, E.E. Boos, L.V. Dudko, Single top quark at future hadron colliders: complete signal and background study. Phys. Rev. D 59, 075001 (1999). arXiv:hep-ph/9806332

53. T.M.P. Tait, The $t W^{-}$mode of single top production. Phys. Rev. D 61, 034001 (2000). arXiv:hep-ph/9909352

54. C.D. White, S. Frixione, E. Laenen, F. Maltoni, Isolating Wt production at the LHC. J. High Energy Phys. 0911, 074 (2009). arXiv:0908.0631 [hep-ph]

55. J. Alwall et al., MadGraph/MadEvent v4: the new Web generation. J. High Energy Phys. 0709, 028 (2007). arXiv:0706.2334 [hep$\mathrm{ph}]$
56. R. Mertig, M. Bohm, A. Denner, FEYN CALC: computer algebraic calculation of Feynman amplitudes. Comput. Phys. Commun. 64, 345 (1991)

57. S. Frixione, Z. Kunszt, A. Signer, Three jet cross-sections to next-to-leading order. Nucl. Phys. B 467, 399 (1996). arXiv:hep-ph/9512328

58. S. Frixione, A General approach to jet cross-sections in QCD. Nucl. Phys. B 507, 295 (1997). arXiv:hep-ph/9706545

59. R.K. Ellis, G. Zanderighi, Scalar one-loop integrals for QCD. J. High Energy Phys. 0802, 002 (2008). arXiv:0712.1851 [hep-ph]

60. R. Pittau, Final state QCD corrections to off-shell single top production in hadron collisions. Phys. Lett. B 386, 397 (1996). arXiv:hep-ph/9603265

61. P. Falgari, P. Mellor, A. Signer, Production-decay interferences at NLO in QCD for t-channel single-top production. Phys. Rev. D 82, 054028 (2010). arXiv:1007.0893 [hep-ph]

62. J. Pumplin, D.R. Stump, J. Huston, H.L. Lai, P.M. Nadolsky, W.K. Tung, New generation of parton distributions with uncertainties from global QCD analysis. J. High Energy Phys. 0207, 012 (2002). arXiv:hep-ph/0201195

63. S. Frixione, E. Laenen, P. Motylinski, B.R. Webber, Angular correlations of lepton pairs from vector boson and top quark decays in Monte Carlo simulations. J. High Energy Phys. 0704, 081 (2007). arXiv:hep-ph/0702198

64. S. Catani, Y.L. Dokshitzer, M.H. Seymour, B.R. Webber, Longitudinally invariant $K_{t}$ clustering algorithms for hadron hadron collisions. Nucl. Phys. B 406, 187 (1993)

65. M. Cacciari, G.P. Salam, Dispelling the $N^{3}$ myth for the $k_{t}$ jetfinder. Phys. Lett. B 641, 57 (2006). arXiv:hep-ph/0512210

66. K. Hamilton, to appear 